\title{
Congenital Lobar Emphysema with Pulmonary Extramedullary Hematopoiesis
}

\author{
Kishor SURYAWANSHI', Dhiraj NIKUMBH' ${ }^{1}$, Sudhir SINGHAVI', Rajshri DAMLE', Nandkumar DRAVID'
}

'Department of Pathology, A.C.P.M. Medical College, DHULE, INDIA, ${ }^{2}$ Consultant Paediatric Surgeon, DHULE, INDIA

\begin{abstract}
Congenital lobar emphysema is a one of the rare variety of congenital abnormality of the lung causing respiratory distress in newborns and infants. Herein, we report a case of congenital lobar emphysema in a 3-week-old female admitted to the pediatric intensive care unit with severe respiratory distress. CT scan revealed a hyperinflated, hyperlucent left upper lobe, collapse of the ipsilateral lung and marked mediastinal shift to the right. The patient underwent emergency left upper lobectomy with improvement of her condition in the postoperative period. Histopathological findings confirmed the clinical diagnosis of congenital lobar emphysema with an unusual finding of pulmonary extramedullary hematopoiesis. Congenital lobar emphysema often presents a diagnostic and therapeutic dilemma and therefore requires a high index of suspicion in neonates presenting with respiratory distress to avoid morbidity and mortality.
\end{abstract}

Key Words: Extramedullary hematopoiesis, Emphysema, Pneumonectomy

\section{INTRODUCTION}

Congenital lobar emphysema (CLE) is a relatively uncommon congenital lung malformation characterized by severe progressive hyperinflation of one or more pulmonary lobes, compression and collapse of the remaining lung parenchyma and mediastinal shift to the opposite side (1). It is an important cause of respiratory distress in the neonatal and infantile period with a high level of mortality and can result in serious morbidity and disability. CLE is a rare congenital malformation with a prevalence of 1 in 20000 to 30000 (2). Radiological investigations including chest X-ray and CT scan of the thorax are diagnostic modalities.

Though rare, clinical awareness of this entity is important for early diagnosis and effective surgical treatment in neonates and infants presenting with respiratory distress.

\section{CASE REPORT}

A 3-week-old female child weighing $2.8 \mathrm{~kg}$ and from a low socioeconomic status family was referred to the pediatric intensive care unit with severe respiratory distress. The patient's relatives gave a history of repeated episodes of difficulty breathing, poor feeding and fever on and off since birth. She was treated with antibiotics, antipyretics and nebulization therapy for pneumonitis but there was only partial improvement each time. Her respiratory distress had increased for the last 10-12 days and she was referred to the Pediatric Intensive Care Unit for further management. The baby had been born at term by normal vaginal delivery at a primary health center and weighed $2.5 \mathrm{~kg}$ at birth. There

(Turk Patoloji Derg 2017, 33:74-76)

Received : 16.10.2014 Accepted : 08.11.2014 was a history of delayed crying. She had been vaccinated with oral polio vaccine and BCG. On admission, the general examination of the baby showed severe respiratory distress with a respiratory rate of $90 / \mathrm{min}$, heart rate of 140 / min, and temperature of $39.5^{\circ} \mathrm{C}$. The baby was cyanosed and intercostal retraction was present. Respiratory system examination revealed reduced breath sounds on the left upper chest with hyper-resonant percussion note on the left hemithorax. The trachea was shifted to the right. X-ray of the chest showed a hyperlucent left upper lobe with reduced lung markings, collapse of the left lower lobe and mediastinal shift to right with patchy consolidation in the right middle lobe. CT scan showed hyperinflated, hyperlucent left upper lobe, collapse of ipsilateral lung and marked mediastinal shift to right (Figure 1). The patient underwent emergency left upper lobectomy with improvement of her condition in the postoperative period.

Grossly, the received lobectomy specimen measuring $8.5 \times 6.5 \times 2.0 \mathrm{~cm}$ appeared overinflated. The external surface of lung showed hazy pleura, brownish grey in color at places. The cut section was grayish white with occasional brownish lobules separated by fibrous septae, soft to firm in consistency (Figure 2). Microscopically most of the lung parenchyma revealed lobules with overdistension (dilatation) of alveolar sacs. Alveolar septae were widened and edematous and showed congested blood vessels. Focal areas showed intra-alveolar hemorrhages with pigment laden macrophages. Focal area showed alveolar lumina with proteinaceous material, fibrin strands and foci of extramedullary hematopoiesis consisting of myeloid cells

Correspondence: Kishor SURYAWANSHI

Department of Pathology, A.C.P.M. Medical College, DHULE, INDIA

E-mail: ompathologylab@gmail.com Phone: +91 2562276317 


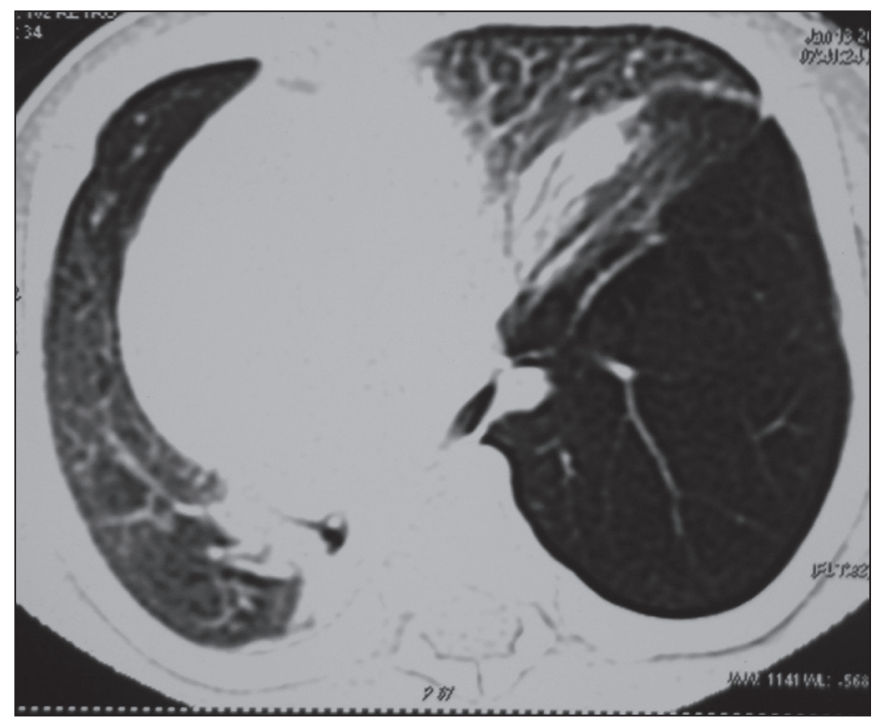

Figure 1: CT scan showed hyperinflated, hyperlucent left upper lobe, collapse of ipsilateral lung, and marked mediastinal shift to right.

and megakaryocytes (Figure 3). The section studied from the left segmental bronchus revealed normal bronchial cartilage and mucus glands.

\section{DISCUSSION}

Congenital lobar emphysema (CLE) was first described in 1932 by Nelson. It is a rare disease with an incidence of 1 in 20000 to 30000 births (2,3). CLE is a one of the rare cystic malformations of lung development characterized by overinflation of the pulmonary lobe. Hyperinflation of alveoli within the pulmonary lobe results in mediastinal shift to the opposite side with compression of the contralateral lung and collapse of the remaining lung (4). $90 \%$ of cases present before 6 months of age but can occur as late as 5 years. Male children are affected more commonly than females (5).

Respiratory distress and dyspnea are the major complaints followed by recurrent respiratory tract infections and cyanosis. A review of the literature showed that the left upper lobe is usually the most frequently affected lobe followed by the middle and right upper lobe, the lower lobes being rarely affected. Okazaki and Kumart reported bilateral involvement of the lung in CLE in their studies $(6,7)$. Definite etiological factors could not be identified in half of the cases. Again in half of the cases, there is partial bronchial obstruction due to absent, hypoplastic or immature bronchial cartilage producing ball valve effect with consequent overinflation, endobronchial obstruction by exuberant mucosal fold, extrinsic compression by aberrant bronchi or vascular structures bronchogenic cyst and rarely congenital CMV infection (8). Recently theory of polyalveosis has been described as a cause of CLE (9). In

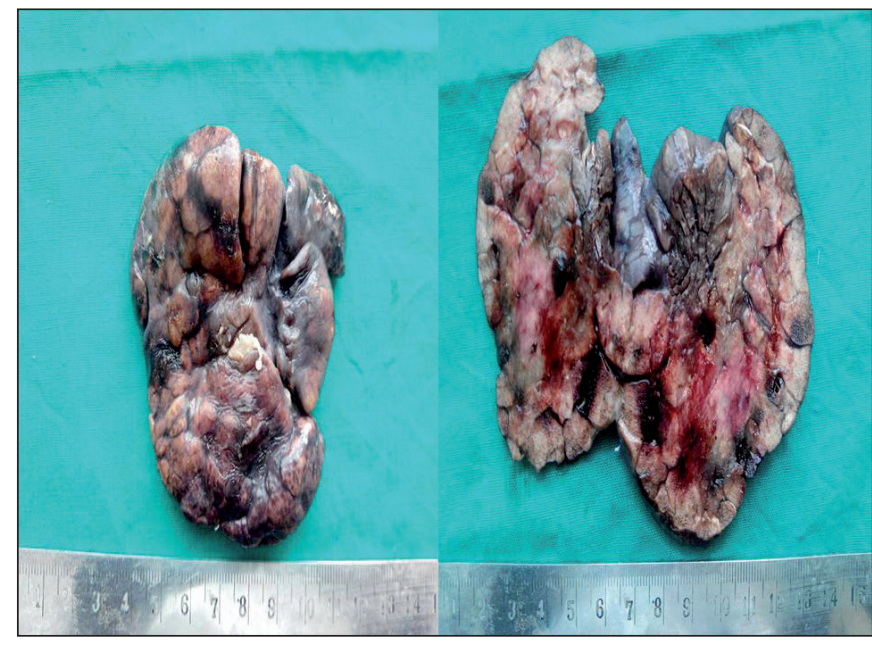

Figure 2: Lobectomy specimen measuring $8.5 \times 6.5 \times 2.0 \mathrm{~cm}$ appears overinflated. External surface of the lung showed hazy pleura, brownish grey at places. The cut section was grayish white, with occasional brownish lobules separated by fibrous septae, soft to firm in consistency.

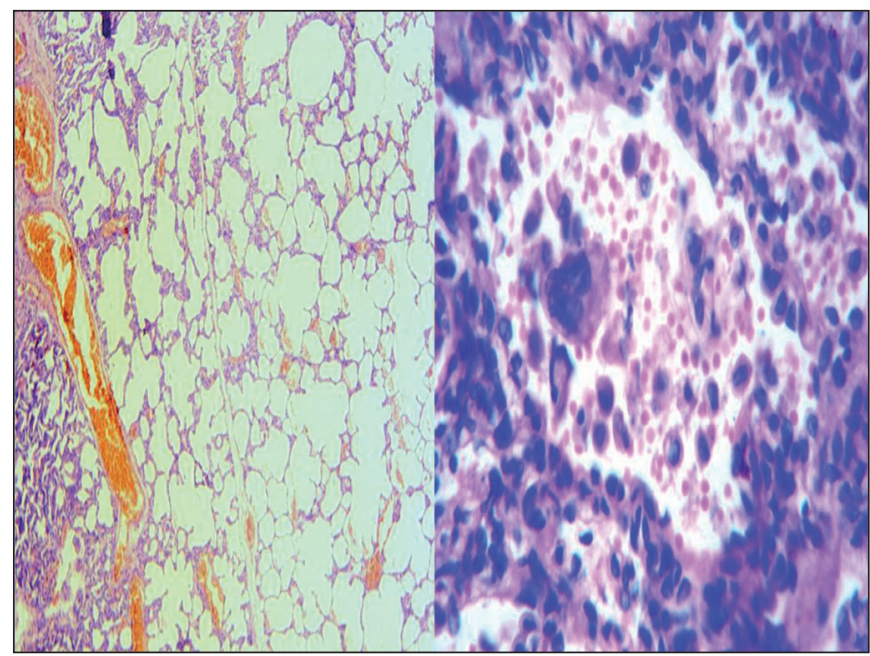

Figure 3: Microscopically most of the lung parenchyma revealed lobules with overdistension (dilatation) of the alveolar sacs. Alveolar septae were widened and edematous and showed congested blood vessels. Focal areas showed alveolar lumina with fibrin strands and foci of extramedullary hematopoiesis consisting of myeloid cells and megakaryocytes (H\&E; x400).

$20 \%$ cases, CLE is known to occur with other malformations and especially cardiac malformations (5). The bronchial cartilage was normal in our case. A definite etiological factor could not be identified in our case as reported in various studies and other malformations including cardiac were absent.

Extramedullary hematopoiesis (EMH) is a compensatory phenomenon in hematological diseases with ineffective hematopoiesis, either due to bone marrow replacement or 
hemolytic anemia with ineffective erythropoiesis. EMH occurs in the liver, spleen and less frequently in the lymph nodes, lungs, serosal surfaces and the urogenital system (10). Pulmonary EMH has been reported in the literature due to myeloproliferative disorders, hemolytic anemia, hereditary spherocytosis and Gaucher's disease (11). Ineffective erythropoiesis forces expansion of the hematopoietic tissue outside the marrow and leads to hematopoietic compensatory involvement, mostly in the form of masses in other regions of the body. A review of the literature revealed congenital cystic adenomatoid malformations (CCAM) with EMH in lung but association of CLE with EMH has not been reported (12). Our case had CLE with EMH without association of any secondary pathology, the cause of which could not be find out. The presumptive pathogenetic mechanism and possible cause of EMH may be chronic hypoxia due to nonfunctional hyperinflated lung compressing surrounding lung parenchyma and release of cytokines that recruit progenitor cells to the lung causing EMH.

The differential diagnosis includes pneumothorax, cysts, and diaphragmatic hernia that can be ruled out by the presence of linear bronchovascular and alveolar markings on chest $\mathrm{x}$-ray. Other causes include pulmonary sequestration, CCAM and upper airway obstruction that can be ruled out by ultrasonography, Color Doppler imaging and magnetic resonance imaging. Prenatal diagnosis of CLE is rare, possibly because of either its low prevalence in utero or the increased echogenicity of the lungs that could be too subtle to be appreciated in utero (13).

Acute severe respiratory distress in association with upper respiratory tract infection occurs in $12 \%$ of patients requiring emergency surgical intervention (1). Surgical excision of the emphysematous lobe is the treatment of choice in severe respiratory distress with a mortality rate less than $5 \%$. Mild cases may be managed conservatively with follow-up of the patient. Surgically treated patients may have abnormal pulmonary function test results but remain asymptomatic with normal growth and development.

Review of the literature showed two recent studies of case series of CLE. In one study, 18 CLE patients were followedup over a period of 10 years. Male preponderance (55.5\%) with varying degrees of respiratory distress (44.4\%) and left upper lobe involvement were the commonest findings in this study. No associated congenital anomaly was detected (14). In another study, 20 patients were followed-up over a period of 30 years at two university hospitals. Equal sex distribution was present while respiratory distress and left upper lobe involvement were the commonest findings in this study. Eighteen patients were treated by lobectomy while in two patients bilobectomy was performed, in conjunction with bronchogenic cyst resection in one case. There were no cartilaginous changes or other etiological factors except for a bronchogenic cyst compressing the upper lobe in one case (5).

Our patient was a female child who presented in a neonatal period with intermittent and progressive respiratory distress. Clinical features were confused with pneumonitis in the early period and pneumothorax later on but characteristic chest $\mathrm{x}$-ray and CT scan findings ruled out these conditions. Emergency left upper lobectomy was performed in view of the severe respiratory distress. Histopathological findings confirmed CLE with an unusual finding of extramedullary hematopoiesis.

To conclude, CLE often presents a diagnostic and therapeutic dilemma and therefore requires a high index of suspicion in neonates presenting with respiratory distress to avoid morbidity and mortality.

\section{REFERENCES}

1. Lierl M. Congenital abnormalities. In: Pediatric respiratory disease: Diagnosis and treatment, Hilman BC. 1st Ed. Philadelphia: WB Saunders Com; 1993.468-72.

2. Thakral CL, Maji DC, Sajwani MJ. Congenital lobar emphysema: Experience with 21 cases. Pediatr Surg Int. 2001;17:88-91.

3. Nelson RL. Congenital cystic disease of the lung: Report of a case. J Pediatr. 1932;1:233-8.

4. Paramesh H. Textbook of practical pediatric pulmonology. 4th ed. Philadelphia: McGraw-Hill; 2009. 70-9.

5. Cataneo DC, Rodrigues OR, Hasimoto EN, Schmidt Jr AF, Cataneo AJ. Congenital lobar emphysema: 30 -year case series in two university hospitals. J Bras Pneumol. 2013;39:418-26.

6. Tadaharu O, Sumio K, Naoto U, et al. Management of bilateral congenital lobar emphyseam. Japanese Journal of Pediatric Surgery. 2002;34:1380-5.

7. Sathish KT, Anna S, Sudipta S. Bilateral congenital emphysema. Unusual cause for recurrent respiratory tract infection. JIAPS. 2006; 11:151-3.

8. Olutoye OO, Coleman BG, Hubbard AM, Adzick NS. Prenatal diagnosis and management of congenital lobar emphysema. J Pediatr Surg. 2000; 35:792-5.

9. Guidici R, Leao LE, Moura LA, Wey SB, Ferreira RG, Crotti PL. Polyalveolosis: Pathogenesis of congenital lobar emphysema? Rev Assoc Med Bras. 1998; 44:99-105.

10. Dingli D, Utz JP, Krowka MJ, Oberg AL, Tefferi A. Unexplained pulmonary hypertension in chronic myeloproliferative disorders. Chest. 2001;120:801-8

11. Bowling MR, Cauthen CG, Perry CD, Patel NP, Bergman S, Link KM, Sane AC, Conforti JF. Pulmonary extramedullary hematopoiesis. J Thorac Imaging. 2008;23:138-41.

12. Kuga T, Esato K, Kaneko J, Yamagata Y, Hoshii Y, Takahashi M. Congenital cystic adenomatoid malformation with extramedullary hematopoiesis of the lung: A case report. Journal of Pediatric Surgery. 1997;32:1751-3.

13. Quinton AE, Smoleniec JS. Congenital lobar emphysema-the disappearing chest mass: Antenatal ultrasound appearance. Ultrasound Obstet Gynecol. 2001;17:169-71.

14. Hussen WM, Alkawaz SSA, Abood LS. Congenital lobar emphysema. Egypt J Surg. 2013;32:116-20. 